\title{
Editorial
}

\section{Logic and logistics: conundrum in reperfusion treatment for acute myocardial infarction}

The key to survival in patients with acute myocardial infarction is rapid, complete, and durable myocardial reperfusion. The past two decades have witnessed impressive improvements in the ability to provide such treatment via pharmacological thrombolysis or mechanical restoration of flow. As such, a "good problem to have" has evolved: how to choose between two very effective strategies? While there is relatively little argument that small subsets of patients benefit particularly from primary angioplasty (significant contraindications to fibrinolysis, cardiogenic shock), for the vast majority of patients the two treatments offer comparable benefit. Choosing between the two is frequently a moot point for physicians who do not have immediate and continuous access to invasive therapy, but what influences a physician's decision when both strategies are available remains unclear.

In this issue, Zahn et al attempt to refine our understanding of this decision process by examining the frequency of use of fibrinolysis and primary angioplasty among eight hospitals capable of providing both interventions for patients with acute myocardial infarction eligible for reperfusion treatment. ${ }^{1}$ Of 1532 patients treated at these centres from 1994 to $1997,641(\sim 42 \%)$ received fibrinolysis and $387(\sim 25 \%)$ underwent primary angioplasty. The rest $(\sim 32 \%)$ did not receive reperfusion therapy, despite lack of clear contraindications. Overall, in this non-randomised registry cohort, death $(8.3 \% v$ $13.7 \%)$, reinfarction $(3.9 \%$ v $5.9 \%)$, and heart failure $(4.9 \%$ v $16.4 \%)$ occurred substantially less often in the angioplasty group than in the fibrinolysis group. Patients who did not receive any reperfusion treatment had, as expected, the worst outcome. The rate of use of primary angioplasty varied widely among the centres, from $1.8 \%$ in the hospital enrolling a third of the cohort to $58 \%$ in the second highest enrolling centre. Approximately equal numbers of patients were admitted during office and nonoffice hours (defined as after 17:00 or on weekends). Analysing the effect of time of admission on the choice of therapy, the authors showed that in all hospitals surveyed, most lytic administration occurred during "off-hours" (50-69\%), regardless of the overall use of primary angioplasty in that centre. In other words, even if a hospital treated most patients with primary angioplasty (hospitals 1 and 5), they preferred to use lytics at night and angioplasty during the day. This dichotomy was particularly relevant to hospitals with a lower use of primary angioplasty, which did not provide consistent 24 hours a day availability. Delay to each treatment by time of admission and breakdown of the proportion of patients admitted during off-hours at each hospital were not provided. Theoretically, the wide range of angioplasty use could be explained by a significant imbalance in the "on" and "off" hours rates of admission at each institution. The study conclusions were that "discretion of the individual hospital" and time of admission influence treatment selection, while patient characteristics do not.

What is "hospital discretion" and how do these observations compare with existing data?

\section{Randomised trials and registries of reperfusion treatment}

Weaver et al compiled data from 10 randomised studies of primary angioplasty versus fibrinolysis in over 2600 patients. $^{2}$ By 30 days from randomisation, there was a substantial reduction in the incidence of death (34\%), and death and reinfarction $(42 \%)$ among angioplasty patients. In contrast, the myocardial infarction triage and intervention (MITI) registry reported identical rates of death among $\sim 3000$ patients treated, in a non-random manner, with angioplasty $(\mathrm{n}=1095)$ or fibrinolysis $(n=2095)$ in the Seattle area. ${ }^{3}$ Zahn et al's study presents data more in line with randomised studies with respect to outcome, although the absolute event rates were twice as high as reported in the meta-analysis by Weaver et al.

While fibrinolysis is typically available around the clock within 30-60 minutes of arrival at hospital, delays to primary angioplasty experienced during off-hours may explain the predominance of lytic use during that interval in this study and at many other institutions. Cannon et al examined the relation between delay to angioplasty and outcome in the national registry of myocardial infarction (NRMI) $2^{4}$ and found that in-hospital mortality increased from $6.9 \%$ among those treated within 60 minutes of presentation to $9.1 \%$ for those with a delay of $>2$ hours. Unfortunately, more than $50 \%$ of patients followed in this registry had a $>2$ hour delay to angioplasty. Anderson et al analysed outcome of patients treated with either reperfusion strategy and concluded that the expected mortality in patients receiving fibrinolysis within 25 minutes of presentation or angioplasty within 75 minutes of presentation is $6.3 \%$ and $5.8 \%$, respectively, ${ }^{5}$ very similar to the results of the largest randomised study of angioplasty versus lytics (GUSTO-IIb angioplasty substudy; global utilisation of streptokinase and tissue plasminogen activator for occluded coronary arteries). ${ }^{6}$

Furthermore, the economic impact of establishing continuous availability of invasive services is not negligible, as demonstrated by Lieu et al in their theoretical cost analysis. ${ }^{7}$ In the US, a hospital admitting at least 200 patients with acute myocardial infarction each year $(40 \%$ undergo primary angioplasty) and offering primary angioplasty 24 hours a day, would spend US $\$ 1597$ per procedure. In contrast, implementation of new night technical cover, under similar baseline assumptions, would increase the cost to at least US\$3206 per procedure.

\section{Clinical implications}

When performed in a timely fashion by experienced operators, primary angioplasty provides superior arterial patency and improves outcome compared with fibrinolysis. The increasingly wider utilisation of stents ${ }^{89}$ and potent platelet glycoprotein IIb/IIIa receptor antagonists ${ }^{10}$ further extends this benefit. Thus, when available promptly, angioplasty is often the optimal strategy of reperfusion. The preferential utilisation of angioplasty during on-hours in the MITRA study ${ }^{1}$ conforms to this logic. Conversely, the wider use of lytics during off-hours reflects the logistics of primary angioplasty, including difficulty in providing around the clock cover without exaggerated delays, and covering the costs of human and material resources. 
Thus, it appears that hospital discretion, as presented by Zahn et al integrates the logic and logistics of reperfusion treatment in a non-randomised setting, selecting the strategy that can offer the best outcome in a given set of circumstances.

Can the two converge in a superior strategy that achieves better patency than fibrinolysis at a lower risk of major haemorrhage and without the delays inherent to primary angioplasty? The answer will emerge, probably, from the concept of combined platelet and fibrin lysis, as currently tested in a large clinical study, the GUSTO IV-AMI. Platelet IIb/IIIa integrin blockade in combination with low dose fibrinolytics can achieve TIMI 3 flow (thrombolysis in myocardial infarction) in about $80 \%$ of patients, often obviating the need for immediate angioplasty, which can be performed safely at a later time, in most patients, in a semielective manner. Further comparison of this "facilitated" approach to mechanical reperfusion after combination platelet and fibrin lysis, with the conventional primary angioplasty strategy, with adjunctive platelet $\mathrm{IIb} / \mathrm{III}$ receptor blockade is scheduled to begin later this year in a 2000 patient international trial. Logic and logistics may ultimately coexist.

$S$ BRENER

E J TOPOL

Department of Cardiology, The Cleveland Clinic Foundation,

9500 Euclid Ave, F-25, Cleveland, OH 44195, USA

email:Topole@ccf.org
1 Zahn R, Schiele R, Seidl K, et al, for the MITRA Study Group. Spectrum of reperfusion strategies and factors influencing the use of primary angioplasty in patients with acute myocardial infarction admitted to hospitals with the facilities to perform primary angioplasty. Heart 1999;82:420-5.

2 Weaver WD, Simes RJ, Betriu A, et al. Comparison of primary coronary angioplasty and intravenous thrombolytic therapy for acute myocardial infarction: a quantitative review . $7 A M A 1997 ; 278: 2093-8$.

3 Every NR, Parsons LS, Hlatky M, et al. A comparison of thrombolytic therapy with primary coronary angioplasty for acute myocardial infarction. Myocardial Infarction Triage and Intervention Investigators. $N$ Engl $\mathcal{F}$ Med 1996;335:1253-60.

4 Cannon CP, Lambrew CT, Tiefenbrunn AJ, et al. Influence of door-toballoon time on mortality in primary angioplasty results in 3,648 patients in the second national registry of myocardial infarction (NRMI-2) [abstract]. f Am Coll Cardiol 1996;27:61A.

5 Anderson JL, Karagounis LA, Muhlestein JB. Explaining discrepant mortality results between primary percutaneous transluminal coronary angioplasty and thrombolysis for acute myocardial infarction [editorial]. Am f Cardiol 1996;78:934-9.

6 The GUSTO IIb Angioplasty Substudy Investigators. A clinical trial comparing primary coronary angioplasty with tissue plasminogen activator for acute myocardial infarction. N Engl f Med 1997;336:1621-8.

7 Lieu TA, Lundstrom RJ, Ray T, et al. Initial cost of primary angioplasty for acute myocardial infarction. F Am Coll Cardiol 1996;28:882-9.

8 Antoniucci D, Santoro GM, Bolognese L, et al. A clinical trial comparing primary stenting of the infarct-related artery with optimal primary angioplasty for acute myocardial infarction: results from the Florence randomized elective stenting in acute coronary occlusions (FRESCO) trial. $\mathcal{f}$ Am Coll Cardiol 1998;31:1234-9.

9 Suryapranata H, van't Hof AWJ, Hoorntje JCA, et al. Randomized comparison of coronary stenting with balloon angioplasty in selected patients with acute myocardial infarction. Circulation 1998;97:2502-5.

10 Brener SJ, Barr LA, Burchenal JEB, et al, for the RAPPORT Investigators. A randomized, placebo-controlled trial of platelet glycoprotein IIb/IIIa blockade with primary angioplasty for acute myocardial infarction. Circulation 1998;98:734-41. 